\title{
Equivalent Constant Rates for Performance-Based Seismic Assessment of Ageing Structures
}

\author{
Dimitrios Vamvatsikos ${ }^{\mathrm{a}, *}$, Matjaž Dolšek ${ }^{\mathrm{b}}$ \\ ${ }^{a}$ University of Cyprus, Department of Civil and Environmental Engineering, 75 Kallipoleos Str, 1681 Nicosia, Cyprus \\ ${ }^{b}$ University of Ljubljana, Faculty of Civil and Geodetic Engineering Jamova 2, SI 1000 Ljubljana, Slovenia
}

\begin{abstract}
Analytical, closed-form solutions are derived for the computation of equivalent constant rates of limit-state exceedance for structures under seismic loads whose capacity is degrading with time. Seismic guidelines currently designate constant, time-independent probabilities or mean annual frequencies of exceedance that are assumed to remain invariable for the entire design life. These are at odds with the time-dependent, ever-increasing exceedance rates of ageing structures. Based on the concept of social equity and discounting of societal investments, the equivalent constant rate provides a basis for judging the safety of structures with timedependent capacity by allowing comparisons with the code-mandated rates of limit-state exceedance. Starting from the simple SAC/FEMA solution and assuming a power-law degradation of capacity with time and a linear change in the combined epistemic and aleatory variability of capacity, we provide general solutions for the equivalent constant rate and for the limiting case of the average rate over the design life of the structure. The solutions are formulated both in the demand-based and in the intensity-based format, the latter being suitable for all limit-states, even close to global collapse. By using a 7-story reinforced concrete building as an example, we demonstrate the accuracy and the practicality of these approximations for the assessment of an existing structure.
\end{abstract}

Keywords: structures, performance-based earthquake engineering, mean annual frequency, equivalent constant rate, ageing, capacity degradation

\section{Introduction}

The ageing of existing infrastructure has become a major issue in the field of design and analysis. Structures are still being designed without due consideration of the ageing and the weathering that they are subject to. Design codes typically contain only some prescriptive requirements, e.g., mandating a certain thickness for concrete cover or specific corrosion protection measures for steel members. It is simply implied that such measures will let the structure retain the required capacity for the duration of its design life. In a seismic environment, it is precisely this unaccounted for, actual level of capacity that will determine whether a failure will occur or not. Thus, while both the seismic capacity and the weathering of structures have received a lot of research interest, it is the combined effect of the two that matters most in many situations.

On the seismic side, performance-based earthquake engineering (PBEE) has recently emerged to explicitly enable the assessment of structural performance under seismic loads. This is exemplified by the framework adopted by the Pacific Earthquake Engineering Research (PEER) Center to estimate the mean annual frequency (MAF) of exceeding designated limitstates in terms of costs, casualties, downtime or simply structural response (Cornell and Krawinkler [1]). Such approaches

\footnotetext{
${ }^{*}$ Corresponding author

Email address: divamva@ucy . ac.cy (Dimitrios Vamvatsikos)
}

have been greatly simplified by SAC/FEMA [2] that has codified a comprehensive compatible framework based on closedform solutions to estimate the MAF of limit-state exceedance for steel moment-resisting frames. While such significant advances have taken place in this field, they all incorporate the assumption that the structure will remain practically indefinitely in the same initial condition. In other words, the MAFs calculated for the initial state are assumed to be time-independent and to extend to the entire design life of the structure, without any consideration for weathering effects. This is a concept that is integrated in our design methodologies as we typically think of, e.g., the " $10 \%$ in $50 \mathrm{yrs}$ " or the " $2 \%$ in $50 \mathrm{yrs"} \mathrm{exceedance}$ probabilities as typical design objectives for buildings. While the current PBEE approach may provide a comprehensive solution to seismic assessment, it also introduces a large question mark for structures that are subject to weathering effects and their capacity tends to degrade with age.

Such effects may indeed not be critical for structures that are not exposed to severe environments, but they may be detrimental for many others. Recent literature has come to acknowledge this problem, especially in the case of bridges (for example [3-5]). Several ideas have been put forward to deal with such degrading-capacity problems in a rational way, often focusing on the life-cycle cost (e.g., [6]). Unfortunately, applying these methods is not trivial: Estimating life-cycle cost can be an elaborate and difficult operation, especially since one needs to address the cost and the frequency of maintenance schemes. Furthermore, inspection and maintenance actions are not the 
norm for most, if not all, buildings and many bridges. On the contrary, any weathering consideration is typically assumed to be provided via a proper initial design meant to maintain the desired level of safety throughout the design life.

Thus, it becomes attractive to maintain a code-compatible format based on the MAF by reconsidering the typical PBEE approach. When capacity degradation is present, the use of a constant-rate homogeneous Poisson process of limit-state exceedance events is not feasible anymore; the actual rate increases with the age of the structure. Still, it is always useful to derive a constant rate that will, in some reasonable sense, be representative of the ever-changing risk faced by the building over its design life. In other words, we need some way to transform time-dependent rates to the usual time-invariant, constant-rates that are so ingrained in our design philosophy.

The early seeds of such an approach can be found in the definition of the average rate of exceedance proposed, e.g., by Cornell and Bandyopadhyay [7], Amin et al. [8] and also indirectly by Torres and Ruiz [9]. The latter actually provide estimates for the expected number of limit-state exceedance events over a time interval, which, when divided by the interval length, produces the average rate, a useful indicator in itself. Nevertheless, it is not obvious that such indiscriminate averaging is the best solution. For example, from the owner's perspective, exceedance events close to the end of a building's lifetime might not matter as much as events that happen while it is still new.

Seeking a more useful measure, we have turned to the equivalent constant rate (ECR), based on the concept of social equity, as introduced by Yeo and Cornell [10] and applied in [11] for decision-making in an aftershock environment. Here, we shall reformulate the ECR considering the problem of ageing structures under seismic loads and we will focus on the generation of closed-form, simple-to-use expressions based on the popular SAC/FEMA [2] approach.

\section{Definition of ECR for ageing structures}

In order to formulate the ECR for ageing structures we will adapt the work of Yeo and Cornell [10] where it was originally defined for a structure subject to a time-varying aftershock threat. Throughout this section we will closely follow in their footsteps, modifying their derivations and definition to fit our case of time-dependent capacity of ageing structures.

First, let us introduce the concept of "seismic-safety technology", i.e., a repair or rehabilitation scheme that, when implemented on a structure, will improve its performance. Then, for a given structure in a given state at time $\tau=0$ and for a specific limit-state LS, let $Q$ be the amount that, when invested by society into such technology at time $\tau>0$ in the future, it instantaneously prevents the violation of LS due to a seismic event at $\tau$ (Paté [12]). In order to properly account for the time-value of money between $\tau=0$ and the time of LS exceedance, we define $\alpha$ as the discount rate (adjusted for inflation) that is appropriate for societal investments in seismic-safety technologies, having typical values in the range of 3-5\% (see Paté-Cornell [13]). Assuming interest compounding in infinitesimal time-intervals then, if the event is certain to happen at $\tau$, we will need to invest
$Q \mathrm{e}^{-\alpha \tau}$ at time zero. If $\lambda_{\mathrm{LS}}(\tau)$ is the instantaneous rate of LS violations at $\tau$, then the probability of such an event happening in $[\tau, \tau+\mathrm{d} \tau]$ is approximately $\lambda_{\mathrm{LS}}(\tau) \mathrm{d} \tau$. Accordingly, the expected amount that society should invest at time zero to prevent a violation of LS at $\tau$ is approximately $Q \mathrm{e}^{-\alpha \tau} \lambda_{\mathrm{LS}}(\tau) \mathrm{d} \tau$.

For the same structure and limit-state LS and for a given design life, or period of interest, $T_{d}$, we define $\lambda_{\mathrm{LS}}^{\mathrm{ECR}}$ as the timeindependent exceedance rate within $\left[0, T_{d}\right]$ that is "equivalent" to the time-dependent rate $\lambda_{\mathrm{LS}}(\tau)$. Paralleling the definition in [10], "equivalence" means that the expected investment for preventing violation of LS in the future is the same for both cases. Compared to the previous, time-dependent case, the only thing that changes is the probability of an "equivalent" limit-state exceedance event in $[\tau, \tau+\mathrm{d} \tau]$; it is now $\lambda_{\mathrm{LS}}^{\mathrm{ECR}} \mathrm{d} \tau$. Hence, the expected amount of societal investment to prevent a violation of LS around time $\tau$ is now $Q \mathrm{e}^{-\alpha \tau} \lambda_{\mathrm{LS}}^{\mathrm{ECR}} \mathrm{d} \tau$ approximately.

In both of the above cases, i.e., for the time-dependent $\lambda_{\mathrm{LS}}(\tau)$ and the constant $\lambda_{\mathrm{LS}}^{\mathrm{ECR}}$, the total expected investment over the lifetime of the structure can be found by integrating the "instantaneous" amounts over the interval $\left[0, T_{d}\right]$. If, according to our equivalence definition, we equate these totals we get:

$$
\int_{0}^{T_{d}} \lambda_{\mathrm{LS}}^{\mathrm{ECR}} Q \mathrm{e}^{-\alpha \tau} \mathrm{d} \tau=\int_{0}^{T_{d}} \lambda_{\mathrm{LS}}(\tau) Q \mathrm{e}^{-\alpha \tau} \mathrm{d} \tau .
$$

If we cancel out $Q$, integrate the left side and arrange terms to separate $\lambda_{\mathrm{LS}}^{\mathrm{ECR}}$, we reach the following fundamental result:

$$
\lambda_{\mathrm{LS}}^{\mathrm{ECR}}=\frac{\alpha}{1-\mathrm{e}^{-\alpha T_{d}}} \int_{0}^{T_{d}} \lambda_{\mathrm{LS}}(\tau) \mathrm{e}^{-\alpha \tau} \mathrm{d} \tau .
$$

In the sections to follow, we shall develop closed-form approximations to the above equation using two different but equally popular formulations, the $I_{M}$-based and the $E_{D P}$-based. In the meantime, we will briefly discuss the time-independent estimation of $\lambda_{\mathrm{LS}}$ and derive these two formulations.

\section{Estimation of the MAF of limit-state exceedance}

Estimating the mean annual frequency (MAF) of exceeding a certain requirement can be conceptually described using the framing equation adopted by the Pacific Earthquake Engineering Research Center [1, 14],

$$
\begin{aligned}
\lambda\left(D_{V}\right)=\iiint G\left(D_{V} \mid D_{M}\right) \cdot \mid & \mathrm{d} G\left(D_{M} \mid E_{D P}\right) \mid \cdot \\
& \left|\mathrm{d} G\left(E_{D P} \mid I_{M}\right)\right| \cdot\left|\mathrm{d} \lambda\left(I_{M}\right)\right| .
\end{aligned}
$$

To simplify the above equation and the ones to follow, we will loosely use $\lambda(X), f(X), F(X)$, and $G(X)$ to denote the MAF function, probability density function (PDF), cumulative distribution function (CDF), and the complementary CDF (CCDF), respectively, of their arguments. For example, $f(X)$ actually means $f_{X}(x)$ and is a different function from $f(Y) \equiv f_{Y}(y)$.

Actually, Eq. (3) is mostly a conceptual way to explain how the exceedance of a key decision variable $D_{V}$ can be defined 
by breaking up the calculation according to the total probability theorem into smaller, one-step-dependence calculations in consecutive steps that involve the damage measure $D_{M}$, the engineering demand parameter $E_{D P}$ and the intensity measure $I_{M}$. It does not necessarily represent the most practical way to calculate the MAF. As discussed in Vamvatsikos and Cornell [14], if we are not interested in estimating decision variables such as cost, downtime, casualties etc., we can simplify the above equation by using indicator variables for $D_{V}$ and $D_{M}$ to leave only the MAF of exceeding a certain limit-state LS computed on the basis of $E_{D P}$ and $I_{M}$. Actually, in such cases, it will be quite convenient to forego the complex Eq. (3) and try to derive the MAF of limit-state exceedance from first principles. We will take this route, simply to improve understanding of the two basic methods that can be used to calculate the MAF and will be fundamental for the remainder of our development.

Let us start with the hazard curve $\lambda\left(I_{M}\right)$ which represents the MAF of exceeding a certain $I_{M}$ value. At any given $I_{M}$, the rate of $I_{M}$ exceedance in its neighborhood $\left[I_{M}, I_{M}+\mathrm{d} I_{M}\right]$ is approximately constant: $\mathrm{d} \lambda\left(I_{M}\right)$. Thus, "locally" we have a Poisson process of seismic events that exceed the designated level of $I_{M}$. The "local" rate of limit-state LS exceedance $\mathrm{d} \lambda_{\mathrm{LS}}$ defines another Poisson process whose constant rate can be derived from the seismic $I_{M}$-exceedance process by filtering it (e.g., [15]) by the probability of limit-state exceedance for a given $I_{M}$ as

$$
\mathrm{d} \lambda_{\mathrm{LS}}\left(I_{M}\right)=P\left(C<D \mid I_{M}\right) \mathrm{d} \lambda\left(I_{M}\right)
$$

where $C$ is the capacity associated with the limit-state and $D$ the corresponding demand generated by seismic loads. Now the interesting part begins: The probability $P\left(C<D \mid I_{M}\right)$ of limit-state exceedance given the $I_{M}$, often termed the fragility or vulnerability function, can be calculated in two equivalent ways:

$$
P\left(C<D \mid I_{M}\right)=P\left(I_{M c}<I_{M}\right)=F\left(I_{M c} \mid I_{M}\right)
$$

or

$$
P\left(C<D \mid I_{M}\right)=P\left(E_{D P c}<E_{D P} \mid I_{M}\right)
$$

In other words, a structure violates LS if the (random) $E_{D P}$ demand due to earthquake (at a given level of $I_{M}$ ) is above the (random) $E_{D P c}$ capacity of the structure or the $I_{M}$ of the earthquake is above the (random) $I_{M c}$ capacity of the structure. To understand the above formulations it is useful to visualize the cloud of capacity points associated with LS in the $E_{D P}-I_{M}$ plane, each $i$-th point placed at its own $\left(E_{D P c}^{i}, I_{M c}^{i}\right)$ coordinates. For readers familiar with IDAs (Vamvatsikos and Cornell [16]) these can be visualized as one point per single IDA curve, or ground motion record, that signifies the level above which the limit state is violated, both in $E_{D P}$ and in $I_{M}$ terms.

Eq. (5) is straightforward to understand, as $I_{M}$ is given and $I_{M c}$ is a random variable. Thus $P\left(I_{M c}<I_{M}\right)$ is simply the CDF of the capacity $I_{M c}$ (actually the CDF value at the level of $I_{M}$ ), easily estimated from the cloud of capacity points in the $E_{D P}-I_{M}$ plane. For example, we can just make the popular assumption of lognormality and estimate its mean-log and standard deviation of the logs of the $I_{M}$-ordinates of the capacity points. On the other hand, equation 6 is more difficult to apply, as both $E_{D P}$ given $I_{M}$ and $E_{D P c}$ (which is independent of $I_{M}$ ) are random variables. In this case we need to use the total probability theorem to calculate this probability by breaking it up into smaller, more tractable pieces. By conditioning on the value of the $E_{D P}$ :

$$
\begin{aligned}
P\left(C<D \mid I_{M}\right) & =\int_{0}^{+\infty} P\left(E_{D P c}<x \mid E_{D P}=x, I_{M}\right) P\left(E_{D P}=x \mid I_{M}\right) \mathrm{d} x \\
& =\int_{0}^{+\infty} F_{E_{D P c} \mid E_{D P}=x}(x) f_{E_{D P} \mid I_{M}}(x) \mathrm{d} x \\
& =\int_{0}^{+\infty} F\left(E_{D P c} \mid E_{D P}\right) f\left(E_{D P} \mid I_{M}\right) \mathrm{d} E_{D P}
\end{aligned}
$$

where we have dropped the conditioning of $E_{D P c}$ on $I_{M}$, since we consider $E_{D P c}$ a property of the structure and not of the loading level. Similarly to Eq. (5), $F\left(E_{D P C} \mid E_{D P}\right)$ represents the CDF of the $E_{D P}$-capacity, or actually the $\mathrm{CDF}$ value at the level of $E_{D P}$. This distinction becomes helpful in the integrals to follow; therefore we have kept this "conditioning" of $E_{D P c}$ on $E_{D P}$ and $I_{M c}$ on $I_{M}$ in all expressions despite there being no real dependence per se.

In summary, there are two equivalent ways to estimate the probability of exceeding a certain limit-state LS in the $E_{D P^{-}}$ $I_{M}$ plane. The first one is based on the $I_{M}$, and although conceptually simpler it has been traditionally termed the indirect method, (e.g., Shome et al. [17]). The other method, called the direct one, is based on the $E_{D P}$ and needs an additional integration to work properly.

The reason behind this historical naming convention is the apparent difficulty in estimating $P\left(I_{M c}<I_{M}\right)$ when operating outside an IDA setting, since the way timehistory analyses are run is that one prescribes the $I_{M}$ and estimates an $E_{D P}$ response. Assuming that the LS capacity is defined based on the values of response parameters, it seems more natural to express the capacity in $E_{D P}$ terms rather than $I_{M}$, as one should iterate on the prescribed $I_{M}$ value to get the proper responses that will define capacity and ultimately get the proper $I_{M c}$. This is akin to the classic way of estimating response statistics for single-degreeof-freedom oscillators as ductility $\mu$ given the force reduction $R$-factor, which needs no iteration, or as $R$-factor given the ductility $\mu$, which requires iterations on $R$. Actually, in an IDA setting such iterations can be easily removed by clever postprocessing [14], thus equating the computational needs of both approaches. Therefore, since the original thinking behind these names is no longer as intuitive as it used to be, we will henceforth name them according to the variable that they are based on, as for example was done by Jalayer [18]. Thus, method 1 (the indirect) will be the $I_{M}$-based and method 2 (the direct) the $E_{D P}$-based.

Actually, in theory both of the above methods are equivalent. There is no theoretical advantage in using one or the other. Still, in practice, the extra integration needed for the $E_{D P}$-based method, together with the thorny issue of collapse tends to tilt 
the balance towards the $I_{M}$-based way. The problems arise both analytically and numerically when we need to estimate the integral in Eq. (7). When trying to implement closed-form solutions, this immediately becomes a bit of a problem that needs some extra approximations to be analytically integrable. The matters are further complicated due to the eventual collapse of the structure above a certain $I_{M}$-level, where essentially small increases in the $I_{M}$ lead to disproportionately large increases in the response $E_{D P}$, leading to practically "infinite" value of response (e.g., [16]). These values may be difficult to deal with numerically but become even worse analytically, especially in closed form. The reader is directed to Jalayer [18] for a comprehensive treatment of this subject.

In our opinion, it suffices to say that we prefer the $I_{M}$-based method for three basic reasons:

a) The case of "infinite" (or very large) $E_{D P}$-capacity due to global instability is automatically taken care of. While this is not important for serviceability, it needs special attention for near-collapse limit-states in an $E_{D P}$-formulation.

b) There is no need to discuss correlation between demand and capacity (e.g., see Cornell et al. [19]).

c) As we will see later on, a closed-form solution can be derived without the need to approximate the $E_{D P}-I_{M}$ relationship.

With that in mind, let us now discuss the estimation of $\lambda_{\mathrm{LS}}$. For both formulations, this can be done via a simple integration of $\mathrm{d} \lambda_{\mathrm{LS}}\left(I_{M}\right)$ from Eq. (4) over all values of $I_{M}$ :

$$
\begin{aligned}
\lambda_{\mathrm{LS}} & =\int_{0}^{+\infty} \mathrm{d} \lambda_{\mathrm{LS}}\left(I_{M}\right)=\int_{0}^{+\infty} P\left(C<D \mid I_{M}\right) \mathrm{d} \lambda\left(I_{M}\right) \\
& =\int_{0}^{+\infty} P\left(C<D \mid I_{M}\right)\left|\frac{\mathrm{d} \lambda\left(I_{M}\right)}{\mathrm{d} I_{M}}\right| \mathrm{d} I_{M}
\end{aligned}
$$

Note that when changing variables from $\lambda\left(I_{M}\right)$ to $I_{M}$ the limits of integration are inverted: As $I_{M}$ goes to $0, \lambda\left(I_{M}\right)$ goes to $+\infty$ and vice-versa. To retain the original integration limits we can either introduce a negative sign that is canceled out eventually by the negative values of the $\lambda\left(I_{M}\right)$ derivative or take the absolute value of the derivative. As typically done (e.g., [18]), we chose the second option.

Now, depending on the formulation that we choose to represent the conditional probability of limit-state exceedance $P(C<$ $\left.D \mid I_{M}\right)$, we get two different versions of Eq. (8) or, equivalently, two different ways to calculate the same $\lambda_{\mathrm{LS}}$. For the $I_{M}$-formulation we have:

$$
\lambda_{\mathrm{LS}}=\int_{0}^{+\infty} F\left(I_{M c} \mid I_{M}\right)\left|\frac{\mathrm{d} \lambda\left(I_{M}\right)}{\mathrm{d} I_{M}}\right| \mathrm{d} I_{M}
$$

while for the $E_{D P}$-based one:

$$
\lambda_{\mathrm{LS}}=\int_{0}^{+\infty} \int_{0}^{+\infty} F\left(E_{D P c} \mid E_{D P}\right) f\left(E_{D P} \mid I_{M}\right) \mathrm{d} E_{D P}\left|\frac{\mathrm{d} \lambda\left(I_{M}\right)}{\mathrm{d} I_{M}}\right| \mathrm{d} I_{M} .
$$

These equations have appeared in several forms in the past (e.g., see Bazzurro et al. [20] and references therein) and they are the same as the ones found in Vamvatsikos and Cornell [14], the only difference from the latter being that we derived them from first principles rather than transforming the PEER-integral appearing in Eq. (3).

From now and on, we will use, as customary for most applications, $S_{a}$ as the $I_{M}$ and $\theta$ as the $E_{D P}$, thus remaining faithful to the basic derivation by Cornell et al. [19]. It should be noted, though, that different scalar quantities can be used as intensity or response without any problem, as long as the approximations discussed below are respected. Actually, there have been several recent proposals for better scalar $I_{M}$ parameters, e.g., in [21-24], that can improve the reliability of such calculations dramatically.

\section{IM-based formulation}

In order to estimate the ECR via Eq. (2), we first need a closed form solution to Eq. (9). This has been presented by Cornell et al. [19] and explained in detail by Jalayer [18], whose results we will present here. First, we assume that the $I_{M}$-capacity is lognormally distributed and adopt the first-order assumption, i.e., we assume that the epistemic uncertainty does not influence the median value, only the dispersion around the median. This assumption is not entirely accurate, as shown e.g., by Liel et al. [25] and Vamvatsikos and Fragiadakis [26], but still reasonable. Furthermore, we assume that the hazard curve $H(\cdot)$, i.e., the $\lambda\left(I_{M}\right)$ function of mean annual frequency of exceeding values of the $I_{M}$, or $S_{a}$, can be approximated in the area of the median $I_{M}$ capacity, or $\hat{S}_{a, c}$, by a power-law form, or, equivalently, a straight line in log-log coordinates:

$$
\lambda\left(I_{M}\right)=H\left(S_{a}\right)=k_{0}\left(S_{a}\right)^{-k} .
$$

Then, as shown in [18], the mean annual frequency of exceeding a limit-state LS can be approximated as

$$
\lambda_{\mathrm{LS}}=H\left(\hat{S}_{a, c}\right) \exp \left(\frac{k^{2}}{2}\left(\beta_{R S a}^{2}+\beta_{U S a}^{2}\right)\right)
$$

where $\beta_{R S a}$ is the record-to-record dispersion (the standard deviation of the log-data) around the median capacity $\hat{S}_{a, c}$ and $\beta_{U S a}$ represents the corresponding epistemic uncertainty. The reader is referred, for example, to [26, 27] for a comprehensive discussion of how these two quantities, especially $\beta_{U S a}$, can be estimated in the case of model parameter uncertainty.

The typical way that such equations are used is under the assumption that the various parameters, $\hat{S}_{a, c}, \beta_{R S a}, \beta_{U S a}$ are constant over time. Thus, we may consider Eq. (12) as valid for the entire design life of the structure. This is an assumption that provides a very clear meaning to the typical design situations e.g., of " $10 \%$ in 50 years", signifying that for a homogeneous Poisson process with a constant rate of $\lambda_{\mathrm{LS}}$ we can directly connect $\lambda_{\mathrm{LS}}$ to the probability $p=10 \%$ of exceedance over the lifetime $T_{d}=50 \mathrm{yrs}$ as

$$
p=1-\mathrm{e}^{-\lambda_{\mathrm{LS}} T_{d}}
$$


Unfortunately, for the more realistic case of structures subject to environmental actions that slowly degrade their capacity over time, e.g., due to fatigue, corrosion or other processes, the above assumption of a constant rate $\lambda_{\mathrm{LS}}$ may not be adequate. In other words we have to consider $\lambda_{\mathrm{LS}}$ as $\lambda_{\mathrm{LS}}(\tau)$. In such cases, we should treat Eq. (12) as a time-dependent function that is strictly valid only for a given time-instance $\tau$. This is possible as its derivation does not make use of any timeinvariance assumptions, thus making it also useable in a timevariant framework. In the context of Eq. (2), Eq. (12) can actually be thought of as the result of an intermediate or internal integration to derive $\lambda_{\mathrm{LS}}(\tau)$. As discussed in previous sections, it now becomes important to estimate an equivalent constant value that is possible to use just like $\lambda_{\mathrm{LS}}$ in Eq. (13).

\subsection{IM-based equivalent constant rate}

Even with a closed form estimate for $\lambda_{\mathrm{LS}}$, Eq. (2) is not amenable to analytical solution, unless we assume simplified expressions for the, potentially time-dependent, parameters in estimating $\lambda_{\text {LS }}$ via Eq. (12).

First of all, we will assume that $\hat{S}_{a, c}$ does not degrade dramatically. Then, we can use the same approximation for the hazard curve for all times $\tau$. Thus, in the form of Eq. (11) we will assume that the constants $k_{0}$ and $k$ are time-independent. In order to apply this practically, we cannot fit the hazard curve via Eq. (11) just at the $\hat{S}_{a, c}$ point as proposed by Cornell et al. [19]. The problem is the potential overestimation of the hazard rate for low $S_{a}$ 's. Since the hazard rates there are exponentially higher, this is the area with the most significant contribution to the integral in Eq. (9). Actually, as discussed by Vamvatsikos and Cornell [14], both a global fit and a tangential point-fit at a given $\hat{S}_{a, c}$ may sometimes be appropriate and other times in gross error. Extensive testing [28] has shown that a better, more robust approximation can be achieved through a local area-fit. For example, for a given $\hat{S}_{a, c}$ it is better to fit within $\left[0.25 \hat{S}_{a, c}\right.$, $\left.1.25 \hat{S}_{a, c}\right]$, rather than right at $\hat{S}_{a, c}$. This is a good strategy to achieve a robust estimation even just at the $\hat{S}_{a, c}$ point for $\tau=0$, namely $\hat{S}_{a, c}^{0}$, with Eq. (12). As discussed later in the example's section, if we slightly extend this interval around $\hat{S}_{a, c}^{0}$ to cover even lower values then, depending on the degradation rate of the capacity, we can also achieve a good fit for multiple $\hat{S}_{a, c}$ values as $\tau$ grows. Therefore, such a local area-fit strategy helps both ways.

In approximating $\lambda_{\mathrm{LS}}^{\mathrm{ECR}}$, the form of the degradation for the $\hat{S}_{a, c}$ capacity of the structure over time $\tau$ is also important. Initially, we will assume that capacity degradation occurs (or continues) immediately from $\tau=0$, without delay, and it may follow either a linear or a power-law form. Since, the latter choice will make no difference on the approximations we need to employ to make Eq. (2) integrable analytically, we will go with the second and more powerful option. Thus, we assume that

$$
\hat{S}_{a, c}(\tau)=\hat{S}_{a, c}^{0}-\gamma \tau^{\delta}=\hat{S}_{a, c}^{0}\left(1-\frac{\gamma \tau^{\delta}}{\hat{S}_{a, c}^{0}}\right)
$$

where, as stated earlier, $\hat{S}_{a, c}^{0}$ is the median $S_{a}$ capacity at time $\tau=0$. If $\delta=1$ we have the linear degradation case [e.g., 9], while $\delta>1$ denotes an accelerating degradation case and $\delta<1$ a decelerating one. Each of these may be appropriate for different situations and case studies.

Regarding the dispersions $\beta_{R S a}(\tau)$ and $\beta_{U S a}(\tau)$, we may either assume that they are constant or take them to vary linearly with $\tau$. Again, both of these assumptions make no difference for the analytical treatment of Eq. (2), thus we will use the second and more powerful option:

$$
\beta_{R U S a}^{2}(\tau)=\beta_{R S a}^{2}(\tau)+\beta_{U S a}^{2}(\tau)=\beta_{R U S a}^{0}{ }^{2}+c_{\beta} \tau
$$

where

$$
\beta_{R U S a}^{0}=\sqrt{\beta_{R S a}^{0}{ }^{2}+\beta_{U S a}^{0}}
$$

is the combined aleatory and epistemic uncertainty in the median $S_{a}$ capacity at time $\tau=0$. Note that the above formulation allows us to deal with various phenomena that are inherent in ageing. For example, the uncertainty associated with the degradation process may change over time, probably increasing. While at time zero there is no degradation-related uncertainty (assuming that we know the present state of the structure, or no degradation has occurred yet), once it starts its influence will increase with time. The above framework allows us to capture this fundamental effect with relative ease.

By employing the above assumptions, the time-dependent $\lambda_{\mathrm{LS}}(\tau)$ can be written with the use of Eq. (12) as:

$$
\begin{aligned}
\lambda_{\mathrm{LS}}(\tau) & =H\left(\hat{S}_{a, c}(\tau)\right) \exp \left(\frac{k^{2}}{2} \beta_{R U S a}{ }^{2}(\tau)\right) \\
& =k_{0}\left[\hat{S}_{a, c}^{0}\left(1-\frac{\gamma \tau^{\delta}}{\hat{S}_{a, c}^{0}}\right)\right]^{-k} \exp \left(\frac{k^{2}}{2}\left(\beta_{R U S a}^{0}{ }^{2}+c_{\beta} \tau\right)\right) \\
& =\lambda_{\mathrm{LS}}^{0}\left(1-\frac{\gamma \tau^{\delta}}{\hat{S}_{a, c}^{0}}\right)^{-k} \exp \left(\frac{k^{2}}{2} c_{\beta} \tau\right)
\end{aligned}
$$

where

$$
\begin{aligned}
\lambda_{\mathrm{LS}}^{0} & =k_{0}\left(\hat{S}_{a, c}^{0}\right)^{-k} \exp \left(\frac{k^{2}}{2} \beta_{R U S a}^{0}{ }^{2}\right) \\
& =H\left(\hat{S}_{a, c}\right) \exp \left(\frac{k^{2}}{2} \beta_{R U S a}^{0}{ }^{2}\right)
\end{aligned}
$$

is the rate of limit-state exceedance $\lambda_{\mathrm{LS}}$ at time zero, i.e., at the start of the period of interest $T_{d}$.

At its present form, Eq. (17) cannot be integrated analytically unless $k$ is an integer. Even then, the resulting expression will depend on $k$ 's value. Thus, we need to approximate the term $(\cdot)^{-k}$ with a simpler form. When the rate of degradation is relatively close to linear, as observed in several cases (e.g., [9, 29]), then we can use a single-parameter exponential function:

$$
\mathrm{e}^{\phi \tau} \approx\left[1-\frac{\gamma \tau^{\delta}}{\hat{S}_{a, c}^{0}}\right]^{-k}
$$

$\phi$ can be estimated through regression or, simply, by matching the two functions at times $\tau=0$ and $\tau=\rho T_{d}$, where $0<\rho \leq 1$. Since the fit at $\tau=0$ is guaranteed by the exponential form 


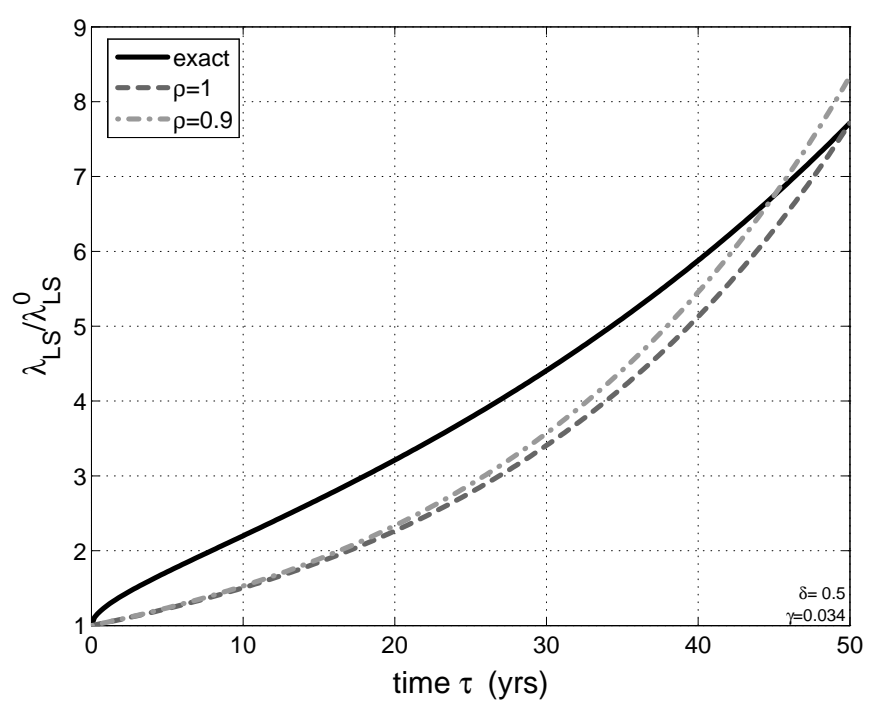

(a) $\delta=0.5$

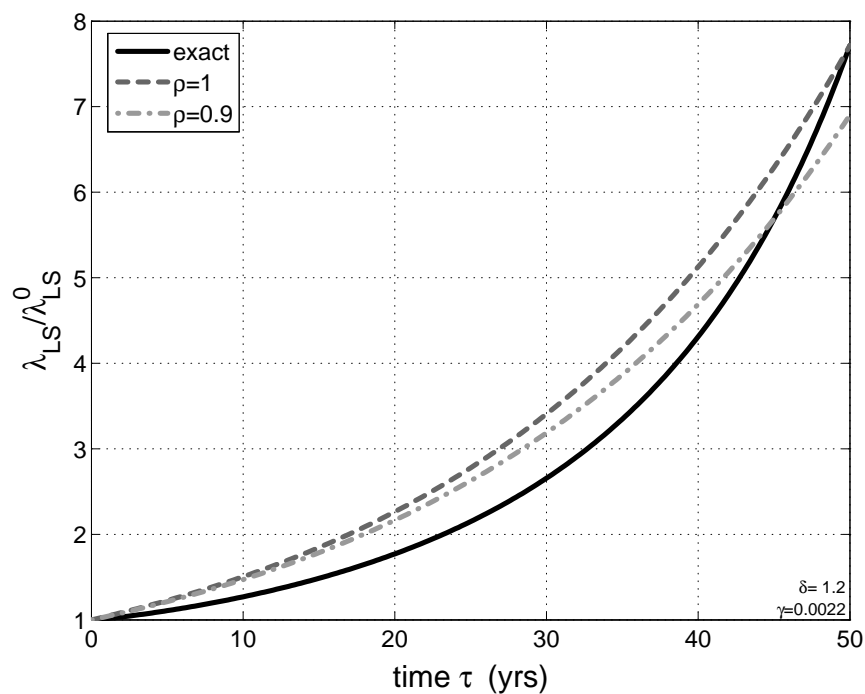

(c) $\delta=1.2$

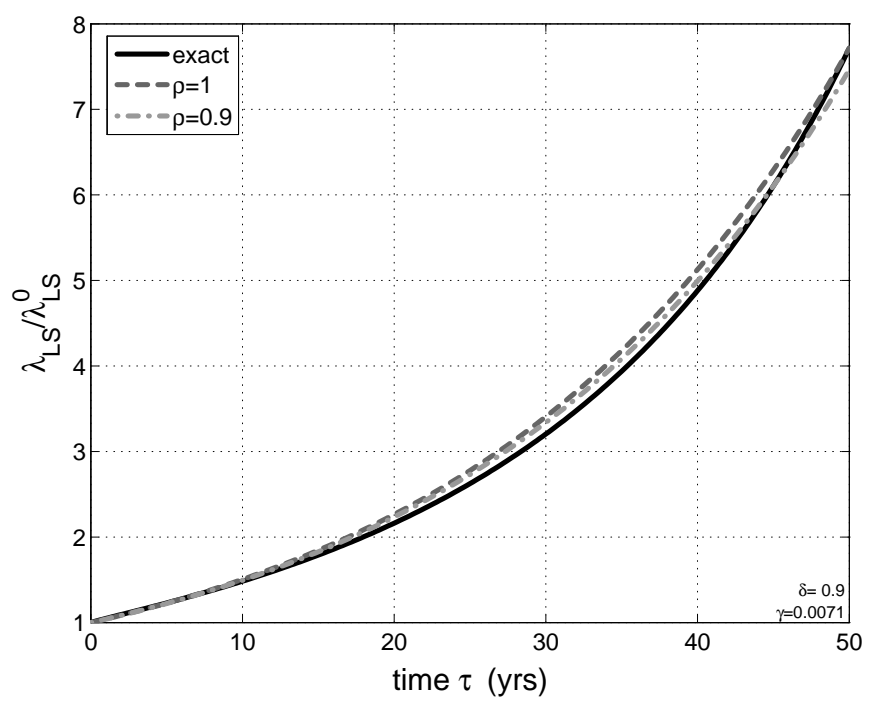

(b) $\delta=0.9$

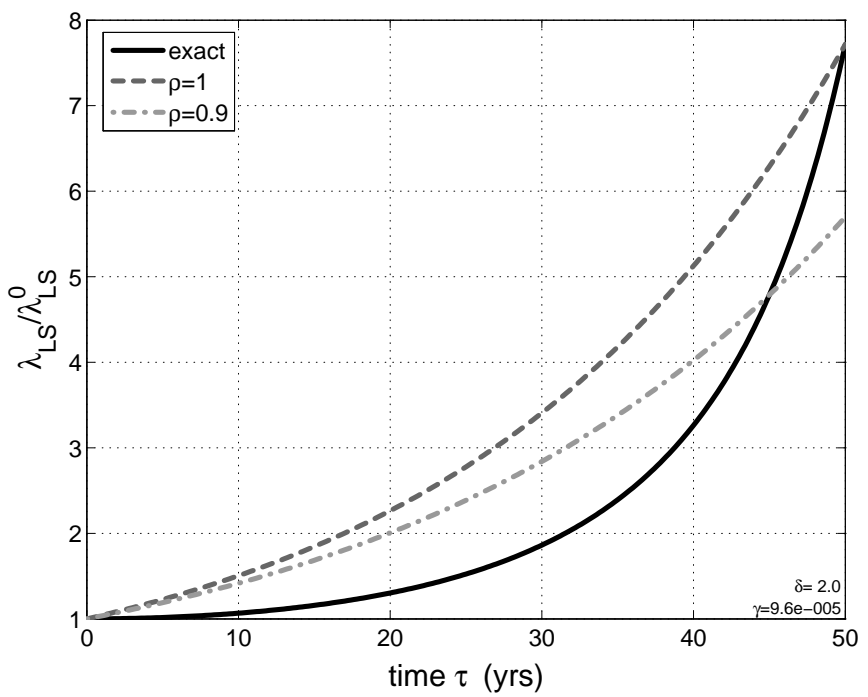

(d) $\delta=2.0$

Figure 1: The exponential approximation to the $(\cdot)^{-k}$-term for different values of $\delta$ for $\hat{S}_{a, c}(50 y r s)=0.6 \hat{S}_{a, c}^{0}$. Values $\delta>2.5$ and $\delta<0.4$ may introduce errors, especially if the $\hat{S}_{a, c}$ degrades to low values. The $\rho=0.9$ approximation is more accurate but the $\rho=1$ is slightly conservative in most cases.

itself, we only have one parameter and one condition to fulfill, leading to

$$
\phi=-\frac{k}{\rho T_{d}} \ln \left[1-\frac{\gamma\left(\rho T_{d}\right)^{\delta}}{\hat{S}_{a, c}^{0}}\right] .
$$

The above form has proven to be an accurate approximation when using a value of $\rho \in[0.85,1]$ and assuming the rate of degradation is not too rapid or too slow. Actually, it works best when, within the period of interest $T_{d}, \hat{S}_{a, c}$ will not degrade to less than $50 \%$ of $\hat{S}_{a, c}^{0}$, or in other terms $\gamma T_{d}^{\delta}<0.5 \hat{S}_{a, c}^{0}$, and $\delta$ is not too far from 1 , i.e., $\delta \in[0.4,2.5]$. The first condition is actually a prerequisite of our whole attempt to provide a closed-form solution. Large changes in $\hat{S}_{a, c}$ will invalidate our assumption that the hazard curve can be approximated reliably by a straight line in $\log -\log$ for all values of $\hat{S}_{a, c}$. The second assumption makes sure that the degradation rate of the exponential function can reasonably match the power-law form. As shown in Fig. 1, when these conditions are respected, the fit is generally good.

By introducing the exponential approximation in Eq. (17), the latter becomes:

$$
\lambda_{\mathrm{LS}}(\tau)=\lambda_{\mathrm{LS}}^{0} \exp \left(\frac{k^{2}}{2} c_{\beta} \tau+\phi \tau\right)=\lambda_{\mathrm{LS}}^{0} \exp \left(\phi^{\prime} \tau\right)
$$

with $\phi^{\prime}=\phi+k^{2} c_{\beta} / 2$ representing the rate of degradation. Now, we can easily integrate Eq. (2) to get:

$$
\lambda_{\mathrm{LS}}^{\mathrm{ECR}}=\lambda_{\mathrm{LS}}^{0} \frac{\alpha}{\alpha-\phi^{\prime}} \cdot \frac{1-\mathrm{e}^{-\left(\alpha-\phi^{\prime}\right) T_{d}}}{1-\mathrm{e}^{-\alpha T_{d}}} .
$$

The above equation is a simple, basic formula to estimate the ECR for a structure with a starting rate of limit-state exceedance $\lambda_{\mathrm{LS}}^{0}$ at time $\tau=0$ for a rate of capacity degradation equal to $\phi^{\prime}$ and a societal discount rate $\alpha$. Actually, Eq. (22) is very robust 
numerically and its behavior has a simple interpretation, as it is directly controlled by the difference between the discount rate and the degradation rate:

- If $\alpha>\phi^{\prime}$ then discounting is faster than degradation, and ECR increases sublinearly with time,

- if $\alpha=\phi^{\prime}$ then discounting and degradation are equally fast, and ECR increases linearly with time,

- if $\alpha<\phi^{\prime}$ then discounting is slower and ECR increases superlinearly with time.

Of course, since $\phi^{\prime}$ depends on time as well, albeit less strongly, the above observations are only approximate, yet quite accurate for a reasonable range of years around $T_{d}$.

Note that there seems to be a possibility for numerical instability for the, practically improbable, case of $\alpha=\phi^{\prime}$. Actually, Eq. (22) is numerically stable, thus if we supply values of $\alpha$ and $\phi$ that differ only slightly, but their difference remains away from the region of round-off error, then it will still yield accurate numerical results. Alternatively, we can take its limit as $\alpha \rightarrow \phi^{\prime}$, or integrate again the simplified Eq. (2) to get the result

$$
\lambda_{\mathrm{LS}}^{\mathrm{ECR}}=\lambda_{\mathrm{LS}}^{0} \frac{\alpha T_{d}}{1-\mathrm{e}^{-\alpha T_{d}}},
$$

which partially confirms our above observations on the behavior of $\lambda_{\mathrm{LS}}^{\mathrm{ECR}}$ through Eq. (22).

\subsection{IM-based average rate}

Another way to derive a constant rate for the time period $\left[0, T_{d}\right]$ is by simply averaging the time-dependent $\lambda_{\mathrm{LS}}(\tau)$ within this range. Although this is not as theoretically attractive as the $\mathrm{ECR}$, the average rate $\lambda_{\mathrm{LS}}^{\mathrm{AVG}}$ is simple to define and understand, while under stricter assumptions it is amenable to more accurate analytical solutions. The seeds of the idea of an average rate appear for example in the work of Torres and Ruiz [9] who used an $E_{D P}$-formulation to define the expected number of failures within a given time period, e.g., $\left[0, T_{d}\right]$. If we divide such a result by $T_{d}$, we can obtain the average rate $\lambda_{\mathrm{LS}}^{\mathrm{AVG}}$, a much more intuitive and usable parameter. More formally, we can define this average rate as

$$
\lambda_{\mathrm{LS}}^{\mathrm{AVG}}=\frac{1}{T_{d}} \int_{0}^{T_{d}} \lambda_{\mathrm{LS}}(\tau) \mathrm{d} \tau .
$$

It becomes obvious from the above expression that we can derive $\lambda_{\mathrm{LS}}^{\mathrm{AVG}}$ as the limit of $\lambda_{\mathrm{LS}}^{\mathrm{ECR}}$ for no discounting, i.e., for $\alpha \rightarrow 0$. Thus, the absence of discounting assures that $\lambda_{\mathrm{LS}}^{\mathrm{AVG}}$ is always greater than $\lambda_{\mathrm{LS}}^{\mathrm{ECR}}$ for a given building and time period. To derive an analytical solution, we can use the same set of assumptions as for $\lambda_{\mathrm{LS}}^{\mathrm{ECR}}$ to integrate Eq. (24) or simply take the limit of Eq. (22) for $\alpha \rightarrow 0$. Both lead to the same result:

$$
\lambda_{\mathrm{LS}}^{\mathrm{AVG}}=\lambda_{\mathrm{LS}}^{0} \frac{\mathrm{e}^{\phi^{\prime} T_{d}}-1}{\phi^{\prime} T_{d}}
$$

It suffices to say that in practice, one does not really need the above formula, as applying a very small $\alpha$, say $0.1 \%$, in
Eq. (22) will still yield the same numerical result; it is quite robust.

Finally, it is worthwhile to mention the limited case of $\lambda_{\mathrm{LS}}^{\mathrm{AVG}}$ when we assume linear degradation of $\hat{S}_{a, c}$ (i.e., $\delta=1$ ) and constant $\beta_{R U S a}$ (i.e., $c_{\beta}=0$ ). Then, we can integrate Eq. (24) without the need for the approximation in Eq. (21) to obtain the "exact" result

$$
\lambda_{\mathrm{LS}}^{\mathrm{AVG}}=\lambda_{\mathrm{LS}}^{0} \frac{\hat{S}_{a, c}^{0}}{\gamma T_{d}(k-1)}\left[\left(1-\frac{\gamma T_{d}}{\hat{S}_{a, c}^{0}}\right)^{1-k}-1\right] .
$$

Representative figures of the results from Eqs (22) and (25) appear in Fig. 2, showing that the error in the closed-form approximations highly depends upon the rate and magnitude of $\hat{S}_{a, c}$ degradation with $\tau$. Although this is not realistic for most practical ageing problems, if $\hat{S}_{a, c}$-degradation accelerates or decelerates too fast (i.e., $\delta \gg 1$ or $\delta \ll 1$ ) or it increases too much (i.e., low $\hat{S}_{a, c}\left(T_{d}\right) / \hat{S}_{a, c}^{0}$ ), then the error increases to levels higher than the maximum $25 \%$ shown in Fig. 2(d).

\subsection{Extension to include time of initiation}

Several ageing processes may be considered as acting immediately on the structure, i.e., starting (or continuing) from the time designated as $\tau=0$ and progressing for the entire $T_{d}$ life. Such cases are, for example, fatigue or the corrosion of exposed steel. On the other hand, there is at least one significant case where weathering may not start immediately from the initial state of the structure (designated as time zero) but may take a number of years $T_{i}$ to initiate. This is the typical case of new reinforced concrete structures where there is a certain time needed for carbonation or for the chloride ions to pass through the concrete matrix before reaching the reinforcement and starting off its corrosion [e.g., 30]. This $T_{i}$ depends on various factors, notable among them being the present state of the structure, the environment and the quality and thickness of the concrete cover.

In such cases, we can easily adapt the proposed expressions to account for $T_{i}$, simply by transforming all functions dependent on $\tau$ to use variable $\tau^{\prime}=\tau-T_{i}$ and letting them be applicable only for $\tau^{\prime} \in\left[0, T_{d}-T_{i}\right]$, while remaining constant and equal to their $\tau^{\prime}=0$ value for any $\tau^{\prime} \leq 0$. By separately performing the integration of Eq. (2) in the $\left[0, T_{i}\right]$ and $\left[T_{i}, T_{d}\right]$ intervals:

$$
\lambda_{\mathrm{LS}}^{\mathrm{ECR}}=\frac{\lambda_{\mathrm{LS}}^{0}}{1-\mathrm{e}^{-\alpha T_{d}}}\left\{1-\mathrm{e}^{-\alpha T_{i}}+\frac{\alpha \mathrm{e}^{-\alpha T_{i}}}{\alpha-\phi^{\prime}}\left[1-\mathrm{e}^{-\left(\alpha-\phi^{\prime}\right) T_{d i}}\right]\right\}
$$

with

$$
\begin{aligned}
& \phi^{\prime}=-\frac{k}{\rho T_{d i}} \ln \left[1-\frac{\gamma\left(\rho T_{d i}\right)^{\delta}}{\hat{S}_{a, c}^{0}}\right]+\frac{k^{2} c_{\beta}}{2}, \\
& T_{d i}=T_{d}-T_{i} .
\end{aligned}
$$

Similarly, for the average rate, we can use the same technique to integrate Eq. (24). If for $\tau>T_{i}$ the value of $\hat{S}_{a, c}$ degrades in a power-law form and $\beta_{R U S a}^{2}$ varies linearly, then

$$
\lambda_{\mathrm{LS}}^{\mathrm{AVG}}=\lambda_{\mathrm{LS}}^{0}\left(\frac{T_{i}}{T_{d}}+\frac{\mathrm{e}^{\phi^{\prime} T_{d i}-1}}{\phi^{\prime} T_{d}}\right)
$$




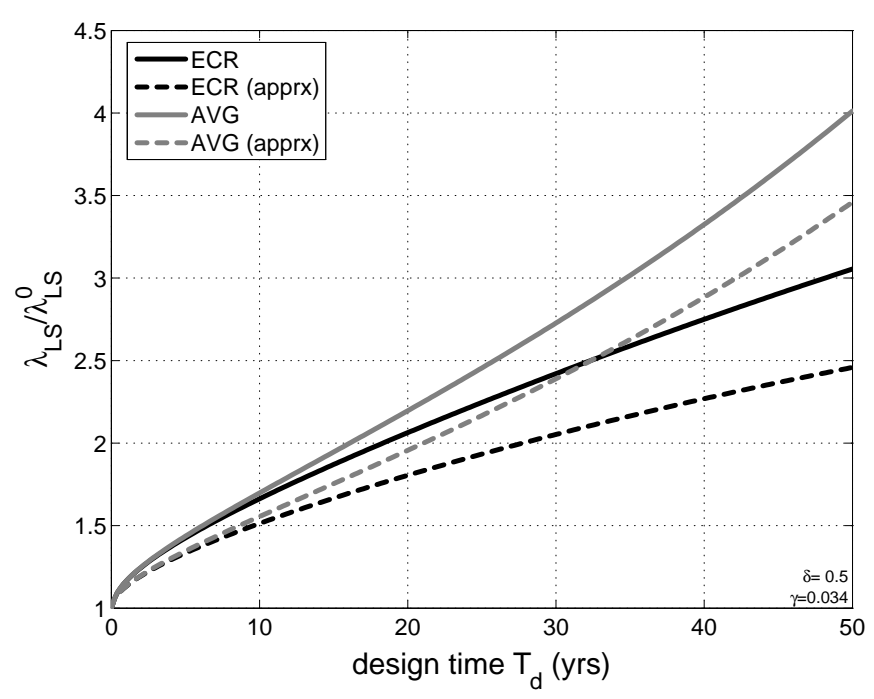

(a) $\delta=0.5$

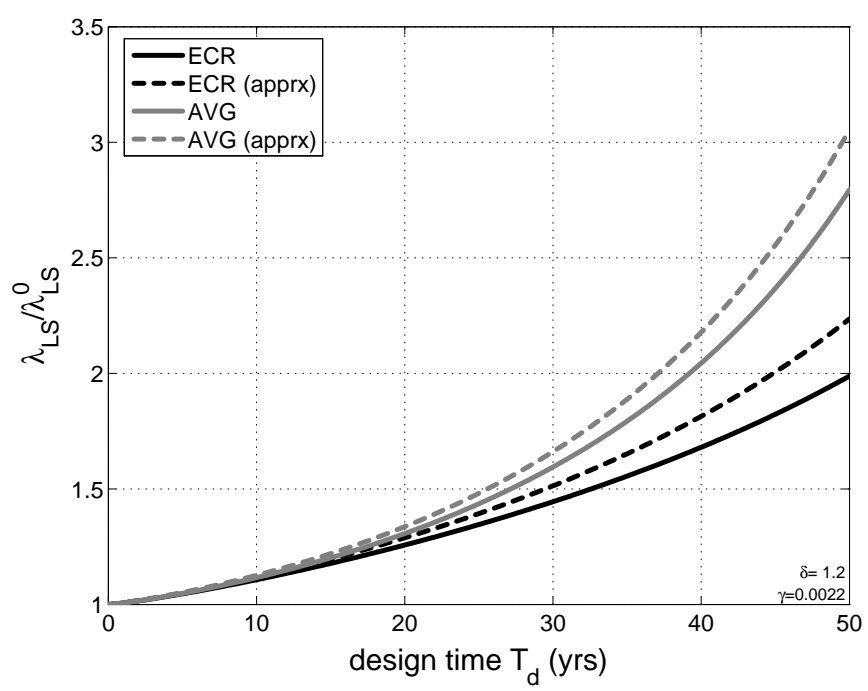

(c) $\delta=1.2$

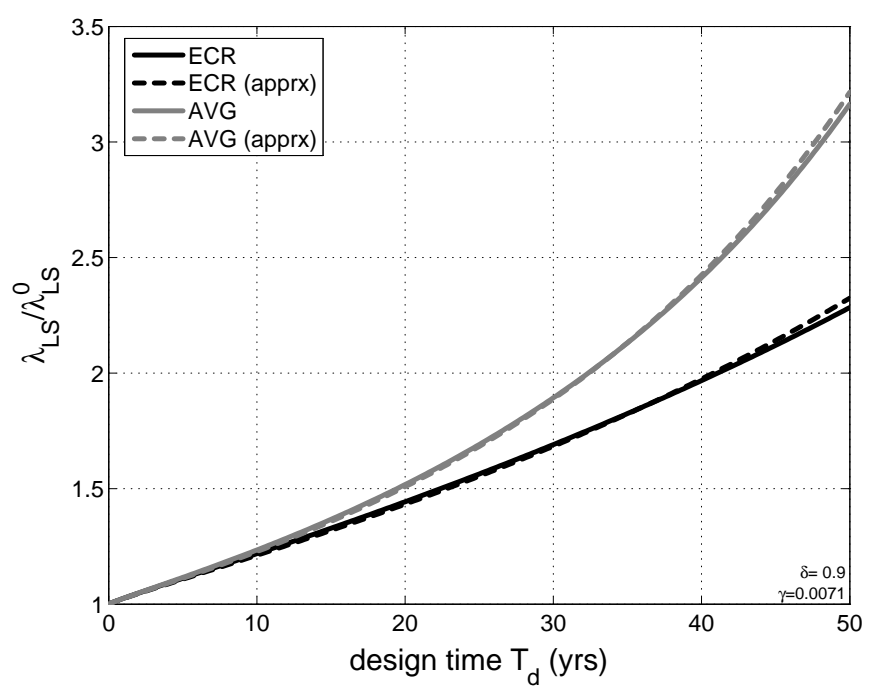

(b) $\delta=0.9$

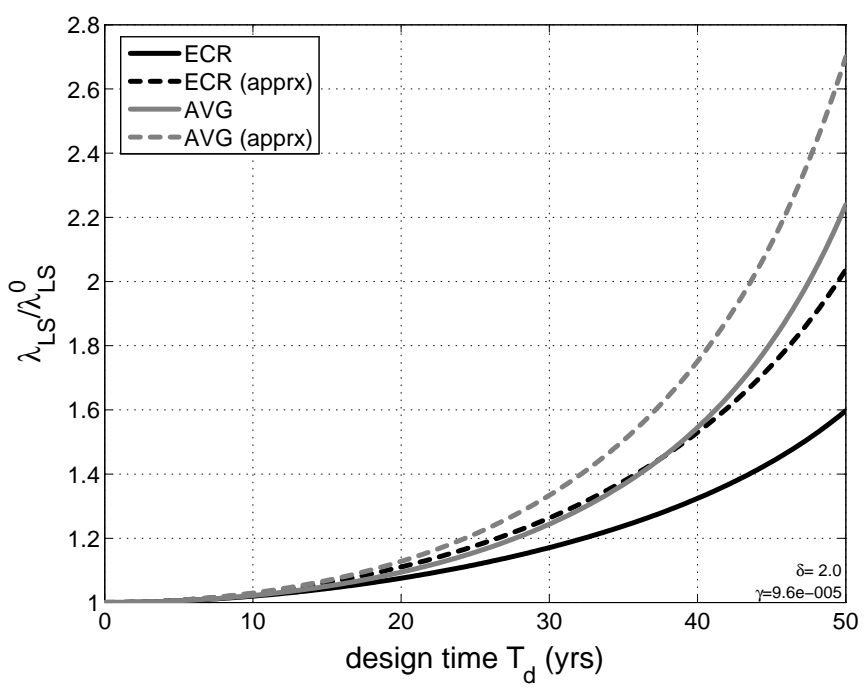

(d) $\delta=2.0$

Figure 2: The exact $\lambda_{\mathrm{LS}}^{\mathrm{ECR}}$ and $\lambda_{\mathrm{LS}}^{\mathrm{AVG}}$ versus their closed-form approximations for $\rho=0.9, \alpha=4 \%$ and various values of $\delta$. The error varies from just $1 \%$ up to $25 \%$ for values of $\delta$ further from 1 and large $\hat{S}_{a, c}$ degradation.

while in the case of linear $\hat{S}_{a, c}$ degradation and constant $\beta_{R U S a}$ we get the analytically "exact" expression:

$$
\lambda_{\mathrm{LS}}^{\mathrm{AVG}}=\lambda_{\mathrm{LS}}^{0}\left\{\frac{T_{i}}{T_{d}}+\frac{\hat{S}_{a, c}^{0}}{\gamma T_{d}(k-1)}\left[\left(1-\frac{\gamma T_{d i}}{\hat{S}_{a, c}^{0}}\right)^{1-k}-1\right]\right\} .
$$

\section{EDP-based formulation}

As discussed in previous sections, $\lambda_{\mathrm{LS}}$ can also be defined on the basis of an $E_{D P}$-formulation. Therefore, using a closedform solution of $\lambda_{\mathrm{LS}}$ in $E_{D P}$-terms, we can develop a $\lambda_{\mathrm{LS}}^{\mathrm{ECR}}$ and $\lambda_{\mathrm{LS}}^{\mathrm{AVG}}$ approximation on the same basis. Actually, Cornell et al. [19] and Jalayer [18] have developed exactly such a solution, where the mean annual frequency of exceeding a limit-state LS can be approximated via an analytical formula if we use some well-known approximations.
First of all we need the approximation of the hazard by a power-law, as expressed by Eq. (11). In addition, we need a power-law approximation of the relationship between the intensity $S_{a}$ and the median response $\hat{\theta}$. This is typically obtained by a linear regression in log-log coordinates and, in the framework of IDA, it can be thought of as an approximation of the median IDA curve:

$$
\hat{\theta} \approx a S_{a}{ }^{b}
$$

A complete description of the several possible ways one can practically estimate the parameters $a$ and $b$ for the above equation can be found in Jalayer and Cornell [31]. For our purposes, it suffices to say that in general $b$ is approximately one for most cases and $a$ can be estimated by a few nonlinear dynamic analyses. Alternatively, they can both be easily estimated through IDA [14]. Still, the approximation needs to be used with care when fitting close to the global-instability region, as discussed 
for example in [18]. In general, the conditioning on no-collapse that is needed to make it work will preclude the derivation of a closed-form solution. Otherwise, as shown in [19], $\lambda_{\mathrm{LS}}$ is found to be:

$$
\lambda_{\mathrm{LS}}=H\left[\left(\frac{\hat{\theta}_{c}}{a}\right)^{\frac{1}{b}}\right] \exp \left(\frac{k^{2}}{2 b^{2}}\left(\beta_{R \theta^{2}}+\beta_{U \theta^{2}}\right)\right)
$$

where $\hat{\theta}_{c}$ is the median $E_{D P}$-capacity and $\beta_{R \theta}, \beta_{U \theta}$ represent the associated record-to-record randomness and epistemic uncertainty dispersions, respectively, for $E_{D P}$ demand and capacity combined. Using this fundamental result, we can derive the $\lambda_{\mathrm{LS}}^{\mathrm{AVG}}$ and $\lambda_{\mathrm{LS}}^{\mathrm{ECR}}$ using similar steps as in the $I_{M}$-formulation for any limit-state that lies away from the near-collapse region.

\subsection{EDP-based equivalent constant rate}

Following our previous derivation, $\lambda_{\mathrm{LS}}^{\mathrm{ECR}}$ is defined according to Eq. (2). To make it amenable to analytical treatment, we need to make similar approximations like for the $I_{M}$-based case. Again, most important is the degradation-law for the $\hat{\theta}_{c}$ capacity of the structure over time $\tau$. We will assume one more time a power-law form

$$
\hat{\theta}_{c}(\tau)=\hat{\theta}_{c}^{0}-\gamma \tau^{\delta}=\hat{\theta}_{c}^{0}\left(1-\frac{\gamma \tau^{\delta}}{\hat{\theta}_{c}^{0}}\right)
$$

where $\hat{\theta}_{c}^{0}$ is the median $\theta$ capacity at time $\tau=0$. Regarding the dispersions $\beta_{R \theta}(\tau)$ and $\beta_{U \theta}(\tau)$, we may either assume that they are constant [e.g., 9], or take them to linearly change with time $\tau$. Again, both of these assumptions make no difference for the analytical integrability of Eq. (2), thus we will use the second option:

$$
\beta_{R U \theta}^{2}(\tau)=\beta_{R \theta}^{2}(\tau)+\beta_{U \theta}^{2}(\tau)=\beta_{R U \theta}^{0}{ }^{2}+c_{\beta} \tau
$$

where

$$
\beta_{R U \theta}^{0}=\sqrt{{\beta_{R \theta}^{0}}^{2}+\beta_{U \theta}^{0}{ }^{2}}
$$

is the combined aleatory and epistemic uncertainty dispersion in the $E_{D P}$ demand and capacity at time $\tau=0$.

Following the same steps that we used before, we arrive at the exact same result of Eqs (22),(27) for zero or non-zero $T_{i}$ respectively. Only the constants change to become

$$
\begin{aligned}
\lambda_{\mathrm{LS}}^{0} & =H\left[\left(\frac{\hat{\theta}_{c}}{a}\right)^{\frac{1}{b}}\right] \exp \left(\frac{k^{2}}{2 b^{2}} \beta_{R U \theta}^{0}{ }^{2}\right) \\
\phi & =-\frac{k}{b \rho T_{d}} \ln \left[1-\frac{\gamma\left(\rho T_{d}\right)^{\delta}}{\hat{\theta}_{c}^{0}}\right] \\
\phi^{\prime} & =\phi+\frac{k^{2} c_{\beta}}{2 b^{2}}
\end{aligned}
$$

where $T_{d i}$ should replace $T_{d}$ in Eq. (38) for non-zero weathering initiation time $T_{i}$.

Each one of our observations from the $I_{M}$-based formula also applies here. As long as we avoid the near collapse region and respect the limits of our approximations, both the $I_{M}$ and the $E_{D P}$ formulations should yield equivalent $\lambda_{\mathrm{LS}}^{\mathrm{ECR}}$ results.

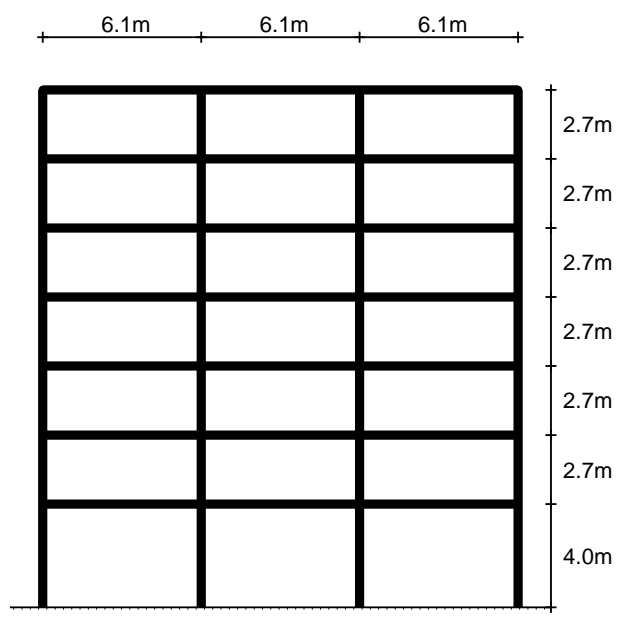

Figure 3: The 7-story Van Nuys reinforced concrete frame studied.

Table 1: The capacity characteristics for the example structure over 50yrs, as determined by IDA for $\tau=0$ and random generation for $\tau>0$.

\begin{tabular}{ccccc}
\hline$\tau(\mathrm{yrs})$ & $\hat{S}_{a, c}(\mathrm{~g})$ & $\beta_{R S a}$ & $\beta_{U S a}$ & $\beta_{R U S a}$ \\
\hline 0 & 1.074 & 0.33 & 0.40 & 0.52 \\
10 & 1.040 & 0.31 & 0.42 & 0.53 \\
20 & 0.988 & 0.30 & 0.46 & 0.55 \\
30 & 0.914 & 0.29 & 0.47 & 0.56 \\
40 & 0.847 & 0.30 & 0.51 & 0.59 \\
50 & 0.795 & 0.28 & 0.53 & 0.60 \\
\hline
\end{tabular}

\subsection{EDP-based average rate}

Similarly to the previous sections, we can derive $\lambda_{\mathrm{LS}}^{\mathrm{AVG}}$ as the limit of $\lambda_{\mathrm{LS}}^{\mathrm{ECR}}$ for no discounting, i.e., as the limit of Eq. (22) for $\alpha \rightarrow 0$. Alternatively, we can directly integrate Eq. (24). In both cases we find the same result as Eqs (25),(30) using of course the constants defined above for the $E_{D P}$-based formulation.

A more accurate analytical result may be derived in the limited case where we assume linear degradation of $\hat{\theta}_{c}$ (i.e., $\delta=1$ ) and constant $\beta_{R U \theta}$ (i.e., $c_{\beta}=0$ ). Then, we can integrate Eq. (24) directly, without the exponential approximation, to obtain:

$$
\lambda_{\mathrm{LS}}^{\mathrm{AVG}}=\lambda_{\mathrm{LS}}^{0} \frac{\hat{\theta}_{c}^{0}}{\gamma T_{d}\left(\frac{k}{b}-1\right)}\left[\left(1-\frac{\gamma T_{d}}{\hat{\theta}_{c}^{0}}\right)^{1-\frac{k}{b}}-1\right],
$$

where $\lambda_{\text {LS }}^{0}$ should be taken from Eq. (37). The case of non-zero $T_{i}$ can be treated in the same way resulting to

$$
\lambda_{\mathrm{LS}}^{\mathrm{AVG}}=\lambda_{\mathrm{LS}}^{0}\left\{\frac{T_{i}}{T_{d}}+\frac{\hat{\theta}_{c}^{0}}{\gamma T_{d}\left(\frac{k}{b}-1\right)}\left[\left(1-\frac{\gamma T_{d i}}{\hat{\theta}_{c}^{0}}\right)^{1-\frac{k}{b}}-1\right]\right\} .
$$

As discussed previously, these equations are similar to the one derived by Torres and Ruiz [9] for the average number of failures in a given period $T_{d}$. To estimate such a number, we only need to multiply $\lambda_{\mathrm{LS}}^{\mathrm{AVG}}$ by $T_{d}$. 


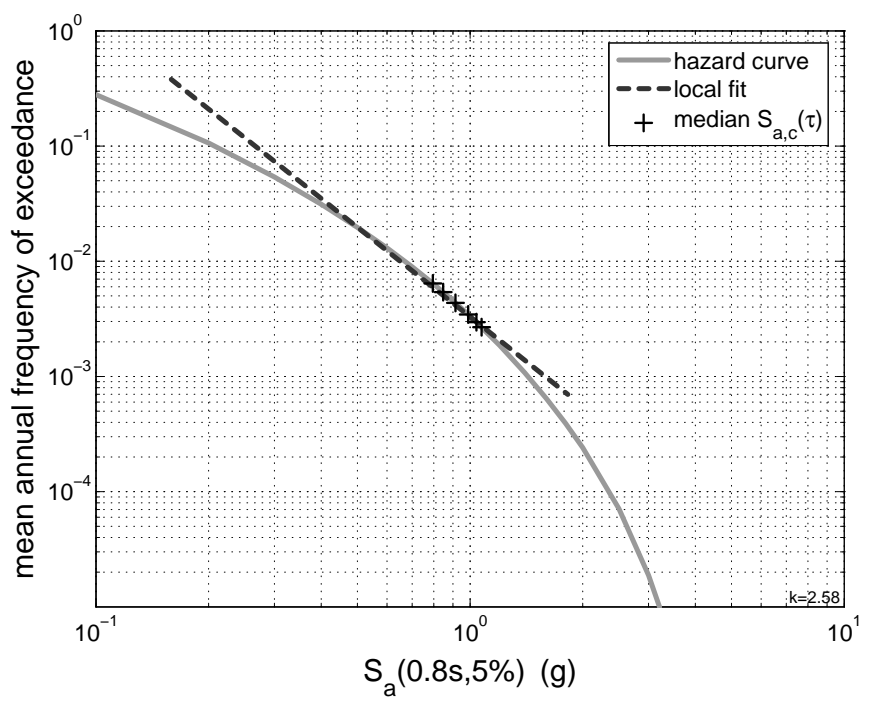

Figure 4: Approximating the hazard curve at $T=0.8$ s by a straight line in $\log$ - $\log$ coordinates.

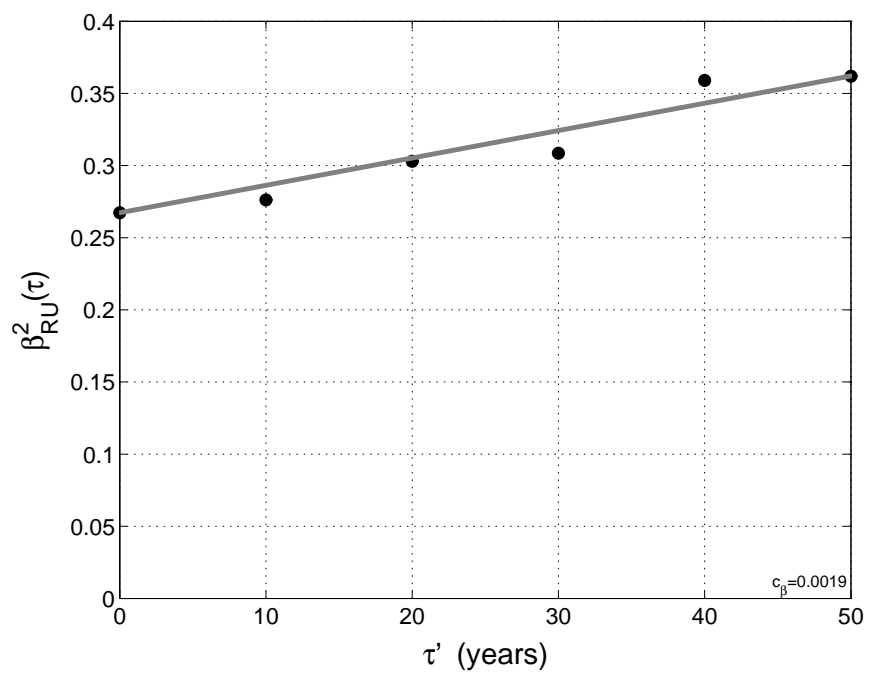

Figure 6: Approximating $\beta_{R U S a}(\tau)$ with a linear fit.

\section{Examples of application}

To show an example of applying the proposed equations on an existing building, we used the transverse frame of the Holiday Inn Hotel in Van Nuys, CA [18]. It is a 7-story hotel located in California's San Fernando Valley, northwest of downtown Los Angeles. The hotel was designed in 1965 according to the 1964 Los Angeles City Building Code, and built in 1966. In plan, the building is rectangular, $19.2 \mathrm{~m}$ by $45.7 \mathrm{~m}, 3$ bays by 8 bays, 7 stories tall (Fig. 3). The structural system is a reinforced concrete moment-frame with flat-plate slabs, but the reinforcing steel lacks ductile detailing.

We have estimated its response and capacity statistics at time zero using IDA [16]. We choose to focus on the Global Instability limit-state, a choice that necessitates the use of the $I_{M^{-}}$ based formulation as discussed earlier. By postprocessing the IDA results [32] we came up with an initial median $S_{a}$-capacity

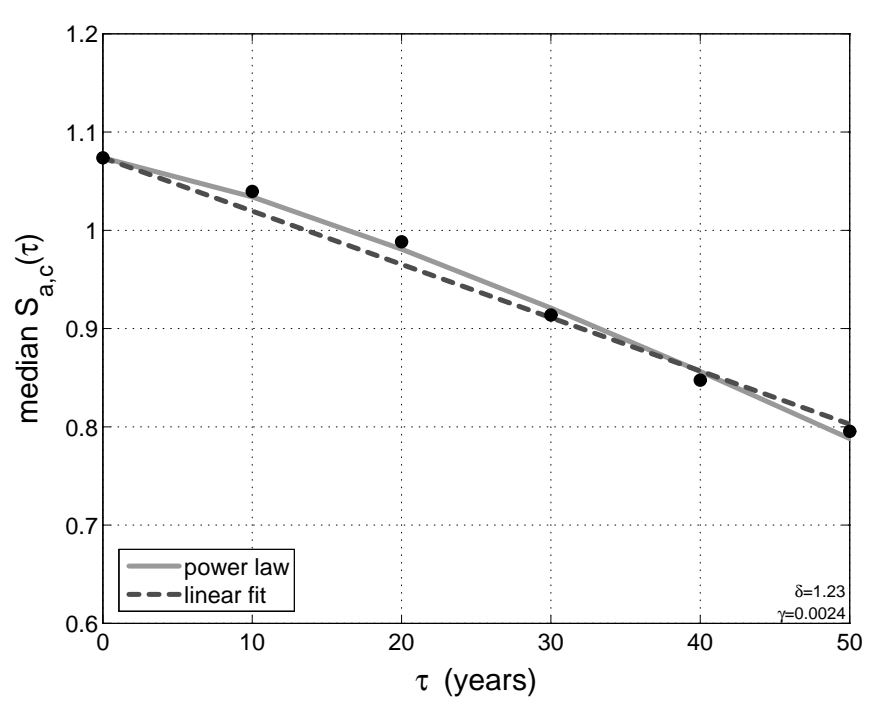

Figure 5: Approximating $\hat{S}_{a, c}(\tau)$ with a linear and a power-law fit.

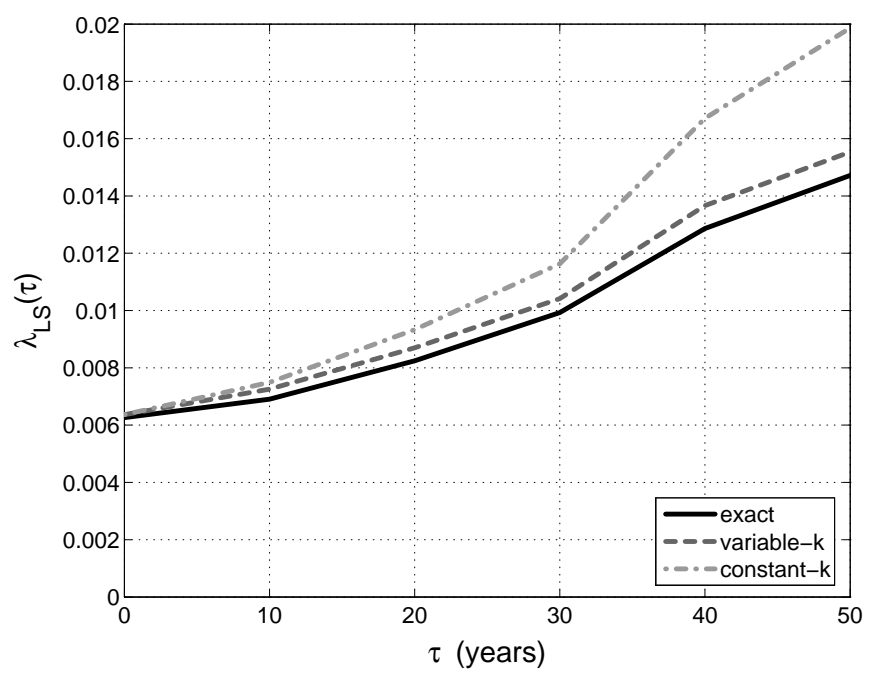

Figure 7: The exact $\lambda_{\mathrm{LS}}(\tau)$ versus its approximations via Eq. (12) assuming a $\tau$-dependent or a constant hazard-curve slope.

of $\hat{S}_{a, c}^{0}=1.07 \mathrm{~g}$ and an associated record-to-record dispersion $\beta_{R S a}=0.33$. In order to calculate the variability in $\hat{S}_{a, c}^{0}$ due to epistemic uncertainties, at least in the model parameters, one can use, for example, the methods in Liel et al. [25], Vamvatsikos and Fragiadakis [26], Dolsek [27]. In our case it was simply assumed that $\beta_{U S a}=0.40$ at time zero.

We should normally repeat the above calculations to determine $\hat{S}_{a, c}, \beta_{R S a}$ and $\beta_{U S a}$ for the corroded state of this structure at several ages of this building within the design life period of $T_{d}=50 \mathrm{yrs}$, for example at times $\tau=10,20,30,40$ and 50 yrs. This entails determining the changes in the structure due to weathering, appropriately modifying the model to account for them and performing IDA to estimate the distribution of limitstate capacity. A comprehensive case-study that actually implements these steps can be found in Celarec et al. [29]. In our case and in order to simplify our example, we decided to simulate the 
degraded state of the building by adopting some deterministic degradation rules for all needed quantities with time $\tau$ and then randomizing by adding some white noise. Thus, we assumed that due to reinforcement corrosion the $\hat{S}_{a, c}$ capacity degrades with time, $\beta_{R S a}$ slightly decreases due to the lower record-torecord variability at lower $S_{a}$-values while $\beta_{U S a}$ increases due to extra epistemic uncertainty, e.g., due to the corrosion process itself. All such degradation processes start immediately from $\tau=0$ since at this time the structure is already several decades old, thus it is reasonable to assume that $T_{i}=0$. The final results appear in Table 1.

Having gathered all the data, we need to follow five discrete steps to determine $\lambda_{\mathrm{LS}}^{\mathrm{ECR}}$ and $\lambda_{\mathrm{LS}}^{\mathrm{AVG}}$, the first two of which are actually the same ones we would use to determine the $\lambda_{\mathrm{LS}}$ of any structure according to the SAC/FEMA guidelines [2]:

a) Fit the hazard curve with a power-law to obtain $k$ [Eq. (11)].

b) Estimate $\lambda_{\text {LS }}^{0}$ [Eq. (18)].

c) Fit $\hat{S}_{a, c}$ vs. $\tau$ with a power-law to get $\gamma, \delta$ [Eq. (14)].

d) Fit $\beta_{R U S a}$ vs. $\tau$ with a straight line to get $c_{\beta}$ [Eq. (15)].

e) Estimate $\lambda_{\mathrm{LS}}^{\mathrm{ECR}}$ and $\lambda_{\mathrm{LS}}^{\mathrm{AVG}}$ [Eqs (22), (25)].

Fitting the hazard curve is probably the most critical step. We need to make sure that the line is fitted in such a way as to be appropriate for all the degraded states of the structure, i.e., for low enough values of $\hat{S}_{a, c}$. Since the structural $S_{a}$-capacity degrades by $20 \%$ at the end of $T_{d}=50 \mathrm{yrs}$, we chose to fit in the region $\left[0.8 * 0.25 \hat{S}_{a, c}^{0}, 1.20 \hat{S}_{a, c}^{0}\right]$. The resulting line appears in Fig. 4 with a slope of $k=2.58$. We used the results to estimate $\lambda_{\mathrm{LS}}^{0}$ at the initial, non-weathered state of the structure. This came out as $\lambda_{\mathrm{LS}}^{0}=0.0064$ via the closed-form solution of Eq. (12), a very accurate result as verified by a numerical integration of Eq. (9) that results to just 0.0063 .

The fitting of the $\hat{S}_{a, c}$ values with time is also simple and it is shown in Fig. 5. Care needs to be exercised, though, when using the power-law form: Fitting a straight line in log-log coordinates via linear regression may not be robust to small changes in $\hat{S}_{a, c}$ for early $\tau$-values. Due to the log-log transformation, the higher changes in $\hat{S}_{a, c}$ that appear later get severely downplayed, sometimes leading to an overall mediocre fit. Thus, it is often better to fit directly in the power-law form via nonlinear regression. This is what we did to find $\gamma=0.0024$ and $\delta=1.23$. In such cases though, especially if $\delta$ is close to 1 , it is simpler and almost as accurate to just fit a straight line in linear coordinates and avoid nonlinear regression. Fig. 5 presents both of the above choices.

Next, we need to fit the epistemic uncertainty $\beta_{R U S a}(\tau)$. We can either use the average $\beta_{R U S a}$ and ignore the changes with time, or perform a linear fit. The results are shown in Fig. 6, where the slope for the second option was determined to be $c_{\beta}=0.0019$.

Finally, we have everything we need to apply Eq. (22). For $\alpha=3 \%$ discounting and over the period of $T_{d}=50 \mathrm{yrs}$, we found $\lambda_{\mathrm{LS}}^{\mathrm{ECR}}=0.0100$. Direct numerical integration of Eq. (2) results to 0.0087 , confirming the above result. If the average rate is sought, it can be calculated via Eq. (25) to find $\lambda_{\mathrm{LS}}^{\mathrm{AVG}}=0.0115$, higher than $\lambda_{\mathrm{LS}}^{\mathrm{ECR}}$ as expected. Alternatively, if we assume a constant $\beta_{R U S a}$ and a linear degradation of $\hat{S}_{a, c}$, we can apply Eq. (26) to find $\lambda_{\mathrm{LS}}^{\mathrm{AVG}}=0.0093$. Both of these values are quite accurate as numerical integration results to 0.0097 . In all cases, the error is in the order of $15 \%$. In general, though, our experience from a range of tests with the same structure shows that the error may reach up to $20 \%$ or $25 \%$, especially when the hazard curve fit lies in its steeper, more rapidly-changing part and the changes in $\hat{S}_{a, c}$ are larger over $T_{d}$. This may be better understood by looking at Fig. 7 where we show the estimates of $\lambda_{\mathrm{LS}}$ found via numerical integration versus the approximations calculated analytically via Eq. (12). The closed-form approximation remains accurate enough when we are allowed to fit the hazard curve locally. Still, as $\hat{S}_{a, c}$ moves away from $\hat{S}_{a, c}^{0}$ with time, the constant $k$-slope assumption that we have made for all $\tau$ will start to hurt us and drive the $\lambda_{\mathrm{LS}}$ estimate away from its true value. Although we never directly estimate $\lambda_{\mathrm{LS}}(\tau)$ for any time other than $\tau=0$, its estimation via a constant $k$ is actually incorporated into our analytical solutions. Nevertheless, in practice we do not expect dramatic changes with ageing, therefore, within such rational limits, the proposed formulas can be considered reasonably accurate for practical use.

In conclusion, as promised from the start, we can now understand the effect of corrosion on the seismic safety of the structure over its design life. If we ignore it for this example, we will determine a mean annual frequency of collapse of $\lambda_{\mathrm{LS}}^{0}=0.0063$, or once every 160 years on average. On the other hand, if we take ageing into account, then, for a societal discounting of $3 \%$, we will get an equivalent mean annual frequency of $\lambda_{\mathrm{LS}}^{\mathrm{ECR}}=0.0087$, or once every 115 years. The almost $20 \%$ decrease in $\hat{S}_{a, c}$ resulted in a roughly $40 \%$ increase in the frequency of limit-state violations. Although such numbers come from a, largely, virtual example and should not be taken at face value, they hint at the potential effects of ageing and showcase our simple and practical way to factor them into the performance-based analysis and design of structures, at least in cases where inspection and maintenance schemes are not considered.

\section{Conclusions}

We have presented closed-form expressions for the estimation of the equivalent constant rate of limit-state exceedance for ageing structures with degrading, time-dependent capacity. Based on the idea of societal investment discounting and social equity, the equivalent constant rate is a time-invariant measure that allows direct comparison with code-mandated values typical of seismic guidelines. Alternatively, another possible but less theoretically-attractive candidate is the average rate which represents the limiting case of no discounting.

For each case, two equivalent formulations are possible. The first is based on the engineering demand parameter, i.e., the response, and while more intuitive it is only useful for limit-states away from global instability. The second is directly based on the intensity measure and is both simpler and more robust to use, being applicable to the full range of structural behavior. 
Using as a basis the SAC/FEMA approximation of the mean annual frequency of limit-state exceedance, simple analytical formulas can be derived using only a few assumptions. Most importantly, as long as the structural capacity does not degrade excessively and the rate of degradation does not change too rapidly with time, the closed-form solutions remain accurate for all practical purposes. Using an ageing 7-story reinforced concrete frame as an example, we present a comprehensive and simple to follow example showing the practicality of our approach.

\section{Acknowledgments}

The authors wish to acknowledge the support of the Cyprus Research Promotion Agency under grant CY-SLO/407/04 and the Slovenian Research Agency under grant BI-CY/09-09-002.

\section{References}

[1] Cornell, C.A., Krawinkler, H.. Progress and challenges in seismic performance assessment. PEER Center News 2000;3(2). [Oct 2009]; URL http://peer.berkeley.edu/news/2000spring/index. html.

[2] SAC/FEMA, . Recommended seismic evaluation and upgrade criteria for existing welded steel moment-frame buildings. Report No. FEMA-351; prepared for the Federal Emergency Management Agency; Washington, DC; 2000.

[3] Tao, Z., Corotis, R.B., Ellis, J.H.. Reliability-based bridge design and life cycle management with markov decision processes. Structural Safety 1994;16(1-2):111-132.

[4] Enright, M.P., Frangopol, D.M.. Service-life prediction of deteriorating concrete bridges. ASCE Journal of Structural Engineering 1998;124(3):309-317.

[5] Stewart, M.G., Rosowsky, D.V.. Time-dependent reliability of deteriorating reinforced concrete bridge decks. Structural Safety 1998;20(1):91109.

[6] Frangopol, D.M., Lin, K.Y., Estes, A.C.. Life-cycle cost design of deteriorating structures. ASCE Journal of Structural Engineering 1997;123(10):1390-1401.

[7] Cornell, C.A., Bandyopadhyay, K.. Should we relax seismic criteria for shorter system exposure times? In: Proceedings of Pressure Vessels and Piping Conference. New York, NY: ASME; 1996,.

[8] Amin, M., Budnitz, R., Cornell, C., Kennedy, R., Olson, D., Tang, H.. Reduced seismic loads for temporary conditions. Nuclear Engineering and Design 1999;192(2-3):167-178.

[9] Torres, M.A., Ruiz, S.E.. Structural reliability evaluation considering capacity degradation over time. Engineering Structures 2007;29:21832192

[10] Yeo, G.L., Cornell, C.A.. Equivalent constant rates for post-quake seismic decision making. Structural Safety 2009;31(5):443-447.

[11] Yeo, G.L., Cornell, C.A.. Post-quake decision analysis using dynamic programming. Earthquake Engineering and Structural Dynamics 2009;38(1):79-93.

[12] Paté, M.. Costs and benefits of seismic upgrading of some buildings in the boston area. Earthquake Spectra 1985;1(4):721-740.

[13] Paté-Cornell, M.. Discounting in risk analysis: capital vs. human safety: structural technology and risk. Waterloo: University of Waterloo Press; 1984.

[14] Vamvatsikos, D., Cornell, C.A.. Applied incremental dynamic analysis. Earthquake Spectra 2004;20(2):523-553.

[15] Benjamin, J.R., Cornell, C.A.. Probability, Statistics, and Decision for Civil Engineers. New York: McGraw-Hill; 1970.

[16] Vamvatsikos, D., Cornell, C.A.. Incremental dynamic analysis. Earthquake Engineering and Structural Dynamics 2002;31(3):491-514.

[17] Shome, N., Cornell, C., Bazzurro, P., J.E.Carballo, . Earthquakes, records, and nonlinear responses. Earthquake Spectra 1998;14(3):469500 .
[18] Jalayer, F.. Direct probabilistic seismic analysis: Implementing nonlinear dynamic assessments. PhD Dissertation; Department of Civil and Environmental Engineering, Stanford University; Stanford, CA; 2003. [Oct 2008]; URL http://www.stanford.edu/group/ rms/Thesis/FatemehJalayer.pdf.

[19] Cornell, C.A., Jalayer, F., Hamburger, R.O., Foutch, D.A.. The probabilistic basis for the 2000 SAC/FEMA steel moment frame guidelines. ASCE Journal of Structural Engineering 2002;128(4):526-533.

[20] Bazzurro, P., Cornell, C.A., Shome, N., Carballo, J.E.. Three proposals for characterizing MDOF nonlinear seismic response. ASCE Journal of Structural Engineering 1998;124:1281-1289.

[21] Cordova, P.P., Deierlein, G.G., Mehanny, S.S., Cornell, C.A.. Development of a two-parameter seismic intensity measure and probabilistic assessment procedure. In: Proceedings of the 2nd U.S.-Japan Workshop on Performance-Based Earthquake Engineering Methodology for Reinforced Concrete Building Structures. Sapporo, Hokkaido; 2000, p. 187206.

[22] Vamvatsikos, D., Cornell, C.A.. Developing effcient scalar and vector intensity measures for IDA capacity estimation by incorporating elastic spectral shape information. Earthquake Engineering and Structural Dynamics 2005;34(13):1573-1600.

[23] Luco, N., Cornell, C.A.. Structure-specific, scalar intensity measures for near-source and ordinary earthquake ground motions. Earthquake Spectra 2007;23(3):357-392.

[24] Mehanny, S.S.F.. A broad-range power-law form scalar-based seismic intensity measure. Earthquake Engineering and Structural Dynamics 2009;31(7):1354-1368.

[25] Liel, A.B., Haselton, C.B., Deierlein, G.G., Baker, J.W.. Incorporating modeling uncertainties in the assessment of seismic collapse risk of buildings. Structural Safety 2009;31(2):197-211.

[26] Vamvatsikos, D., Fragiadakis, M.. Incremental dynamic analysis for estimating seismic performance sensitivity and uncertainty. Earthquake Engineering and Structural Dynamics 2010;39(2):141-163.

[27] Dolsek, M.. Incremental dynamic analysis with consideration of modelling uncertainties. Earthquake Engineering and Structural Dynamics 2009;38(6):805-825.

[28] Dolsek, M., Fajfar, P.. The effect of masonry infills on the seismic response of a four storey reinforced concrete frame-a probabilistic assessment. Engineering Structures 2008;30(11):3186-3192.

[29] Celarec, D., Vamvatsikos, D., Dolsek, M.. Simplified estimation of seismic risk for reinforced concrete buildings with consideration of corrosion over time. Bulletin of Earthquake Engineering 2010;(in review).

[30] Val, D., Stewart, M.G.. Reliability assessment of ageing reinforced concrete structures - current situation and future challenges. Structural Engineering International 2009;19(2):211-219.

[31] Jalayer, F., Cornell, C.A.. Alternative non-linear demand estimation methods for probability-based seismic assessments. Earthquake Engineering and Structural Dynamics 2009;38(8):951-1052.

[32] Vamvatsikos, D., Jalayer, F., Cornell, C.A.. Application of incremental dynamic analysis to an RC-structure. In: Proceedings of the FIB Symposium on Concrete Structures in Seismic Regions. Athens, Greece; 2003, p. $1-12$. 